\title{
Thaumatin-Like Protein-1 Gene (Bx-tlp-1) Is Associated with the Pathogenicity of Bursaphelenchus xylophilus
}

\author{
Fanli Meng,1,2 Yongxia Li,1,2,† Xuan Wang,1,2 Yuqian Feng,1,2 Zhenkai Liu,1,2 Wei Zhang,,1,2 and Xingyao Zhang1,2 \\ ${ }^{1}$ Laboratory of Forest Pathogen Integrated Biology, Research Institute of Forestry New Technology, Chinese Academy of Forestry, Beijing, \\ P.R. China \\ ${ }^{2}$ Co-Innovation Center for Sustainable Forestry in Southern China, Nanjing Forestry University, Nanjing Jiangsu, P.R. China \\ Accepted for publication 4 July 2019.
}

\begin{abstract}
The pine wood nematode Bursaphelenchus xylophilus is a destructive species affecting pine trees worldwide; however, the underlying mechanism leading to pathogenesis remains unclear. In this study, a B. xylophilus gene encoding thaumatin-like protein-1 $(B x-t l p-1)$ was silenced by RNA interference to clarify the relationship between the $B x$-tlp- 1 gene and pathogenicity. The in vitro knockdown of $B x$-tlp- 1 with double-stranded RNA (dsRNA) decreased B. xylophilus reproduction and pathogenicity. Treatments with dsRNA targeting $B x$-tlp- 1 decreased expression by

56.7 at 30 days after inoculation. Additionally, analyses of the cavitation of intact pine stem samples by X-ray microtomography revealed that the xylem cavitation area of pine trees inoculated with B. xylophilus treated with $B x$-tlp- 1 dsRNA was $0.46 \mathrm{~mm}^{2}$ at 30 days after inoculation. Results from this study indicated that the silencing of $B x$-tlp1 has effects on $B$. xylophilus fitness. The data presented here provide the foundation for future analyses of $B x-t l p-1$ functions related to $B$. xylophilus pathogenicity.
\end{abstract} $90 \%$, with the silencing effect maintained even in the F3 offspring. Pine trees inoculated with $B$. xylophilus treated with Bx-tlp-1 dsRNA decreased the symptom of wilting, and the disease severity index was
Keywords: Bursaphelenchus xylophilus, thaumatin-like protein, X-ray microtomography
The pine wood nematode (PWN) Bursaphelenchus xylophilus (Steiner \& Buhrer) (Nickle et al. 1981) is a critical plant-parasitic nematode that is harmful to conifers, particularly pine trees. Pine wilt disease (PWD) caused by B. xylophilus has caused considerable economic losses in Asia (Mamiya 1988; Togashi and Shigesada 2006; Yoshimura et al. 1999) and Europe (Abelleira et al. 2011; Branco et al. 2014; Mota et al. 2009; Vicente et al. 2012), especially in Japan, China, South Korea, Portugal, and Spain. This disease was first detected in 1982 in Nanjing, Jiangsu province, China (Sun 1982). During the past 30 years, PWD has spread to 588 counties in 18 provinces in China. Monochamus spp. beetles (Morimoto and Iwasaki 1972; Ryss et al. 2005) are the major vectors for B. xylophilus because they bore through and feed on more than one pine tree during the course of their lives, thereby introducing nematodes into multiple trees. Infected pine trees usually die rapidly, and there is currently no effective treatment to control the spread of the disease. Despite many studies concerning PWD and the PWN, the pathogenic mechanism of $B$. xylophilus has not been completely elucidated.

Plant-parasitic nematodes exhibit diverse interactions with their host plants to obtain nutrients and supplements important for their development and reproduction (Goellner et al. 2000). Additionally, some pathogenesis-related (PR) proteins that are crucial for plant defense systems are generally produced in higher plants and are involved in biotic stress responses (Hoffmann-Sommergruber 2002). The production of these proteins may be stimulated by pathogens or other external factors. Thaumatin-like proteins (TLPs)

†Corresponding author: Y. Li: lyx020419@caf.ac.cn

Funding: This research was funded by the National Key R\&D Program of China grant 2017YFD0600102.

*The $\boldsymbol{e}$-Xtra logo stands for "electronic extra" and indicates that two supplementary figures are published online.

The author(s) declare no conflict of interest.

C 2019 The American Phytopathological Society belong to the PR-5 family and are widely distributed in most plants and some lower animals. For example, they were first identified in Caenorhabditis elegans (Kitajima and Sato 1999); after which, they were detected in angiosperms, gymnosperms, bryophytes, arthropods (Brandazza et al. 2004; Shatters et al. 2006), and fungi (Grenier et al. 2000; Sakamoto et al. 2006). In a previous study, two B. xylophilus TLPs and one cysteine proteinase inhibitor with sequences that are highly similar to those of plant proteins were identified based on a proteomic analysis (Shinya et al. 2013). Additionally, the similarity between the TLPs from B. xylophilus (BxTLP-1) and Pinus monticola (TLP-S3: ADB97933.1) was confirmed by BLAST and structural analyses of the proteins. Moreover, the sequence of a B. xylophilus TLP was reportedly similar to that of a $P$. massoniana protein (Wang et al. 2014). Furthermore, Meng et al. (2017) cloned the gene encoding the P. massoniana TLP (Pm-tlp) and revealed that its expression is associated with the expression of $B x$-tlp1 from B. xylophilus. The sequence homology between Bx-TLP-1 and Pm-TLP has been confirmed (Meng et al. 2017).

Methods involving RNA interference (RNAi) technology are useful for functionally characterizing genes. A previous study confirmed that dsRNA can induce RNAi in C. elegans (Fire et al. 1998). In nematodes, the uptake of exogenous dsRNA is enhanced by neurostimulants such as octopamine (Bakhetia et al. 2005; Dubreuil 2007; Rosso et al. 2005). Moreover, the effects of immersing nematodes in a solution containing dsRNA were investigated with fluorescein isothiocyanate (FITC), which is a fluorescent dye, as a marker. The dye was observed in the stylet, pharynx, and intestinal tract of nematodes. Because RNAi silencing of a gene that is critical for nematode development can decrease pathogenicity, the biotechnological application of RNAi technology may lead to more effective methods of controlling plant-parasitic nematodes (Gheysen and Vanholme 2007; Sindhu et al. 2009).

Cavitation is a typical pathological feature observed during the development of PWD. As PWD progresses, the pits of tracheids and the ray parenchyma become aspirated, and the epithelial cells surrounding the resin canals are denatured. Therefore, the anatomical deterioration of pine trees infected by PWD can more 
easily permit xylem cavitation (Ikeda and Ohtsu 1992; Utsuzawa et al. 2005). Additionally, X-ray microtomography (micro-CT) has been applied to examine xylem cavitation in intact samples (Cochard and Delzon 2013). Micro-CT provides a spatial resolution of approximately $1 \mu \mathrm{m}$, which is sufficient for analyzing the structure of xylem tracheids and resin canals. Furthermore, micro-CT enables scientists to conduct in-depth studies of plant physiological and pathological phenomena via direct observations (Cochard et al. 2015).

In this study, a B. xylophilus TLP gene ( $B x$-tlp- 1$)$ was targeted by dsRNA to evaluate the influence of silencing the TLP gene on the reproduction and pathogenicity of $B$. xylophilus. Additionally, the cavitation of intact pine samples was examined by micro-CT, thus representing a radiological method of studying PWN pathogenicity. The resulting data may provide the foundation for verifying the interaction between B. xylophilus and host pine trees.

\section{MATERIALS AND METHODS}

Materials. The B. xylophilus NXY61 strain analyzed in this study was isolated from the wood chips of infested $P$. massoniana trees growing in Zhejiang province in China and stored in the Forest Pathogen Integrated Biology Laboratory of the Chinese Academy of Forestry in Beijing, China. Three-year-old $P$. massoniana trees were grown in a greenhouse at $25^{\circ} \mathrm{C}$ with $80 \% \mathrm{RH}$.

Preparation of dsRNA for in vitro RNAi. Double-stranded RNA targeting $B x$-tlp- $l$ was used to evaluate the efficiency of RNAi in B. xylophilus (Table 1). A 467-nucleotide dsRNA corresponding to the gfp gene (Luo et al. 2017) was synthesized as the negative control. The dsRNA sequences were synthesized using the T7 RiboMAX Express RNAi System Kit (Promega, Madison, WI, U.S.A.) with PCR products as templates. The PCR program was as follows: $94^{\circ} \mathrm{C}$ for $3 \mathrm{~min} ; 35$ cycles of $94^{\circ} \mathrm{C}$ for $30 \mathrm{~s}, 55^{\circ} \mathrm{C}$ for $30 \mathrm{~s}$, and $72^{\circ} \mathrm{C}$ for $1 \mathrm{~min}$; and $72^{\circ} \mathrm{C}$ for $10 \mathrm{~min}$. Two ssRNAs were mixed together and incubated at $70^{\circ} \mathrm{C}$ for $10 \mathrm{~min}$ and $25^{\circ} \mathrm{C}$ for $30 \mathrm{~min}$ to synthesize the dsRNAs. The generated dsRNAs ( 2 - $\mu$ l aliquots) were evaluated by $1 \%$ agarose gel electrophoresis.

In vitro RNAi targeting $\boldsymbol{B} \boldsymbol{x}$-tlp-1. We used FITC to assess the efficiency of the dsRNA uptake by nematodes. The PWNs $(\approx 10,000$ individuals comprising a mixture of juveniles and adults) were soaked in a buffer solution consisting of $B x-t l p-l$ dsRNA $(2 \mathrm{mg} / \mathrm{ml})$, FITC $(1 \mathrm{mg} / \mathrm{ml}), 50 \mathrm{mM}$ of octopamine, and $\mathrm{M} 9$ salts $(43.6 \mathrm{M}$ of $\mathrm{Na}_{2} \mathrm{HPO}_{4}, 22 \mathrm{mM}$ of $\mathrm{KH}_{2} \mathrm{PO}_{4}, 2.1 \mathrm{mM}$ of $\mathrm{NaCl}$, and $4.7 \mathrm{mM}$ of $\mathrm{NH}_{4} \mathrm{Cl}$ ) (Urwin et al. 2002). Samples were incubated on a rotator for $36 \mathrm{~h}$ at $25^{\circ} \mathrm{C}$ in darkness. To confirm the target-specific silencing, control samples were prepared with PWNs incubated in the buffer alone and in the buffer supplemented with $g f p$ dsRNA. The PWNs were rinsed five times with $\mathrm{ddH}_{2} \mathrm{O}$ and centrifuged. The nematodes were observed with a Zeiss Axio Imager A1 fluorescence microscope to determine the dsRNA uptake efficiency. The number of surviving nematodes was determined by microscopy following the 36-h treatment. Nematodes were considered dead if their bodies were straight and they did not move, even after mechanical prodding.

To investigate the effects of the silencing of $B x$-tlp- 1 on nematode offspring (F1, F2, F3, F4, and F5 generations), we selected 500 fourth-stage propagative juveniles (J4s) to analyze the $B x$-tlp- 1 expression level after the $B x$-tlp- 1 dsRNA treatment (F0). A total of $500 \mathrm{~J} 4 \mathrm{~s}$ were inoculated with Botrytis cinerea in Petri dishes and incubated at $25^{\circ} \mathrm{C}$ for $96 \mathrm{~h}$ to generate the $\mathrm{J} 4 \mathrm{~s}$ of the next generation (F1). We selected $500 \mathrm{~J} 4 \mathrm{~s}$ to analyze $B x$-tlp- 1 expression and another $500 \mathrm{~J} 4 \mathrm{~s}$ to produce the $\mathrm{J} 4 \mathrm{~s}$ of the next generation (F2). We repeated this method to generate the $\mathrm{J} 4 \mathrm{~s}$ of the third, fourth, and fifth generations (F3, F4, and F5).

To analyze $B x$-tlp- 1 expression in the dsRNA-treated nematodes, total RNA was isolated from the nematodes treated with $B x$-tlp- 1 dsRNA (dsTLP1), $g f p$ dsRNA (dsGFP), and buffer alone (CK). A quantitative real-time PCR (qRT-PCR) assay was performed using the LightCycler 480 II system (Roche Diagnostics Ltd., Switzerland) with TB Green Premix Ex Taq (TaKaRa, Japan). The PCR program was as follows: $95^{\circ} \mathrm{C}$ for $30 \mathrm{~s} ; 40$ cycles of $95^{\circ} \mathrm{C}$ for $5 \mathrm{~s}$; and $60^{\circ} \mathrm{C}$ for $35 \mathrm{~s}$. All samples were analyzed in triplicate, and melting curves were prepared for each data point to assess the specificity of the PCR. The B. xylophilus $\beta$-actin gene was used as an internal control for normalizing the expression data. Details regarding the qRT-PCR primers are provided in Table 1. Relative gene expression levels were calculated based on the cycle threshold changes $(\Delta \Delta \mathrm{Ct})$ and were normalized against the $\beta$-actin expression levels. All experiments were performed in triplicate with three biological replicates.

Effects of silencing $B \boldsymbol{x}$-tlp-1 on $\boldsymbol{B}$. xylophilus reproduction. To investigate the effects of silencing $B x$-tlp- 1 on $B$. xylophilus reproduction, 500 fourth-stage propagative juveniles (J4s) treated with dsRNA were inoculated with $B$. cinerea in Petri dishes and incubated at $25^{\circ} \mathrm{C}$. We collected samples at $4,8,12,16$, and 20 days. Additionally, 500 RNAi-treated B. xylophilus J4s were cultivated in Petri dishes containing $B$. cinerea, after which the PWN populations in each dish were isolated and counted. The population structure was used to evaluate the effects of RNAi-mediated silencing of Bx-tlp-1 on B. xylophilus reproduction.

Pinus massoniana symptoms after various PWN treatments. Three-year-old $P$. massoniana trees grown in a greenhouse were disinfected with $75 \%$ ethyl alcohol and then inoculated with a $300-\mu \mathrm{l}$ suspension comprising $B$. xylophilus $(\approx 3000$ nematods, including juveniles and adults) that had been treated with CK, dsGFP, or dsTLP1. Specifically, $0.5-\mathrm{cm}$-long wounds on the trees at $\approx 40 \mathrm{~cm}$ above the soil level were inoculated and then sealed with Parafilm. Sterile water was used as a control. All treatments were analyzed with three replicates. The inoculated P. massoniana trees were incubated in the greenhouse for 30 days. PWD symptoms and the disease severity index (DSI) were recorded as previously described (Yu et al. 2012). Disease symptoms were assessed based on a scale of 0 to 4 as follows: 0 , all needles were green; 1,0 to $25 \%$ of the needles were discolored and turned yellow; 2, 25 to $50 \%$ of the needles turned yellow; 3,50 to $75 \%$ of the needles turned yellow; and 4, 75 to $100 \%$ of the needles turned yellow. The infection rates and DSI were calculated using the following formulae (Yu et al. 2012):

$$
\begin{gathered}
\text { Infection rate }=\frac{\sum \text { Number of infected trees }}{\text { Total number of trees }} \times 100 \% \\
\text { DSI }=\frac{\sum \text { Number of diseased trees } \times \text { symptom stage }}{\text { Total number of trees } \times \text { highest symptom stage }} \times 100
\end{gathered}
$$

Cavitation analysis by X-ray micro-CT. To detect cavitation in samples, cross-sections of the stems at 2 to $3 \mathrm{~cm}$ below the inoculation sites were examined by X-ray micro-CT (SkyScan 1172; Belgium) at 30 days after inoculations. The X-ray parameter settings

TABLE 1. List of primers used for RNAi experiments

Primer $5^{\prime}-3^{\prime}$

\begin{tabular}{lll}
\cline { 2 - 3 } Gene & \multicolumn{1}{c}{ Sense } & Antisense \\
\hline$d s T L P 1$ & T7 + CGGTATCGGATGTGCTATT & T7 + CTGGTACAAGTCAAGTTCTTC \\
$d s G F P$ & T7 + TGGTCCCAATTCTCGTGGAAC & T7 + CTTGAAGTTGACCTTGATGCC \\
Bx-tlp- 1 & TGTGGCTGACACTTATGG & AGTCGTCGTTGTAGTTGATA \\
$\beta$-actin & TCCGTACCCTGAAGTTGGCTAACC & AAGTGGAGACGAGGGAATGGAACC \\
\hline
\end{tabular}


were $70 \mathrm{kV}$ and $141 \mu \mathrm{A}$, and 1000 images were acquired during the $360^{\circ}$ sample rotation. After three-dimensional reconstruction, the spatial resolution of the image was $2.5 \times 2.5 \times 2.5 \mu \mathrm{m}$ per voxel according to the CTvox and DataViewer programs. The cavitation area was measured using Image $\mathbf{J}$ (National Institutes of Health, U.S.A.) as described on the National Institutes of Health website (https://imagej.nih.gov/ij/). The measurements were completed for three replicates.

Data analysis. All experiments involving PWNs included three replicates, with each treatment replicated five times. The qRTPCR analysis of each sample also included three replicates. Excel 2013 was used to analyze the $B x-t l p-1$ expression and B. xylophilus
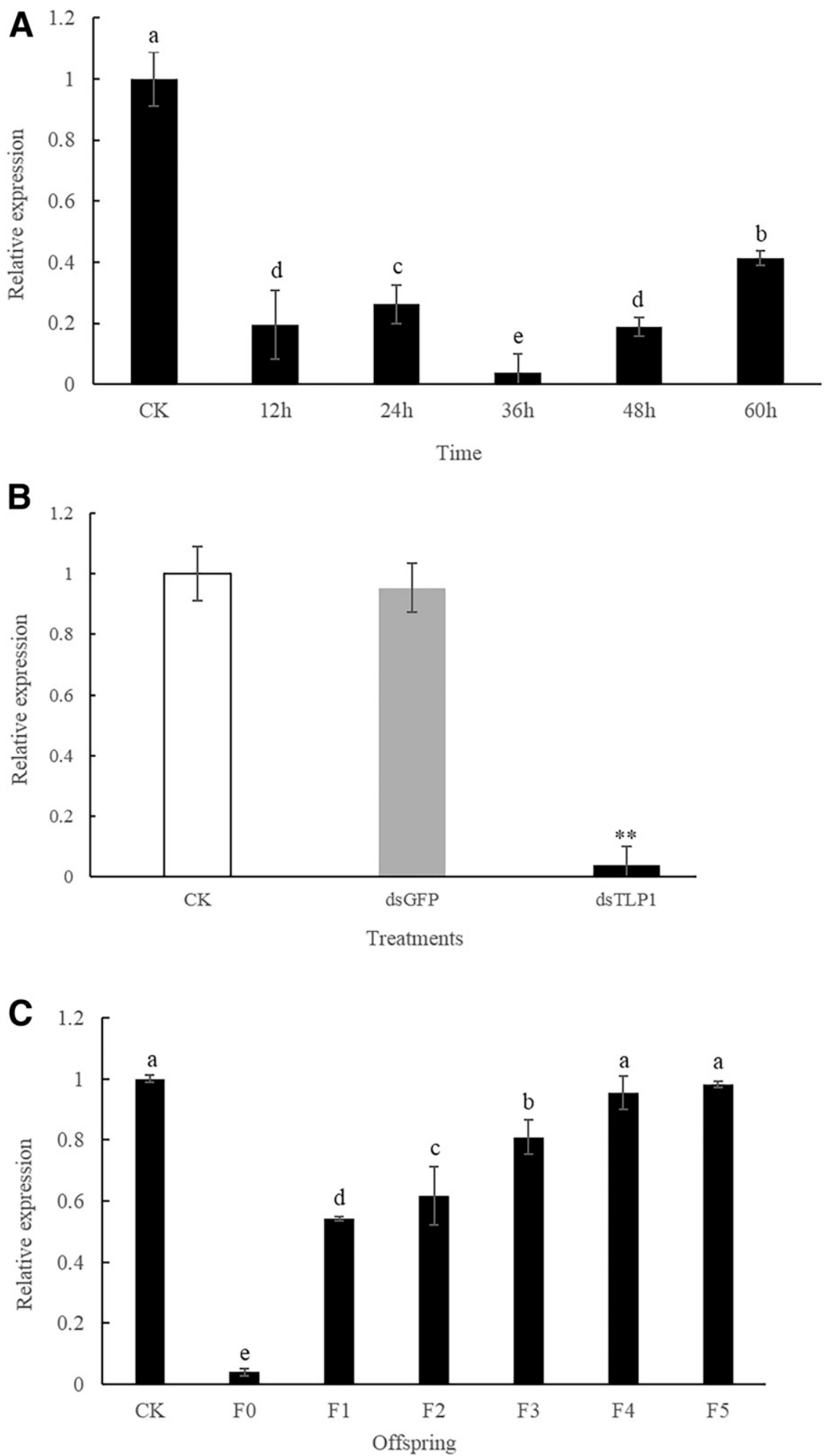

Fig. 1. Effects of RNA interference (RNAi) on Bx-tlp-1 gene expression of Bursaphelenchus xylophilus. The relative expression of the Bx-tlp-1 gene from pine wood nematodes (PWNs) after RNAi for A, different times; B, different treatments; and $\mathbf{C}$, different generations. CK, PWNs treated with the buffer alone; dsGFP, PWNs treated with $g f p$ dsRNA; dsTLP1, PWNs treated with Bx-tlp-1 dsRNA; 12, 24, 36, 48, and $60 \mathrm{~h}$, PWNs treated with $B x-t l p-1$ dsRNA for 12, 24, 36, 48, and $60 \mathrm{~h}$; and F0-F5, F0-F5 generations. Each experiment was repeated three times. The error line means standard deviation of the mean. Bars with different letters indicate significant differences among treatments, as defined by Duncan's test $(P<0.05)$. ** Significant differences among treatments, as defined by $t$ test $(P<0.05)$. 
generations. All data are presented as the mean \pm standard deviation. A single factor analysis of variance (ANOVA) and $t$ test were completed using the SPSS 13.0 program (IBM China Company Ltd., Beijing, China). Differences between samples were considered significant at $P<0.05$.

\section{RESULTS}

In vitro RNAi targeting $B x$-tlp-1 in $B$. xylophilus. To detect the silencing of $B x$-tlp- 1 in vitro, PWNs $(\approx 10,000$ individuals comprising a mixture of juveniles and adults) were treated with a buffer containing dsRNA targeting $B x$-tlp- 1 . A qRT-PCR analysis

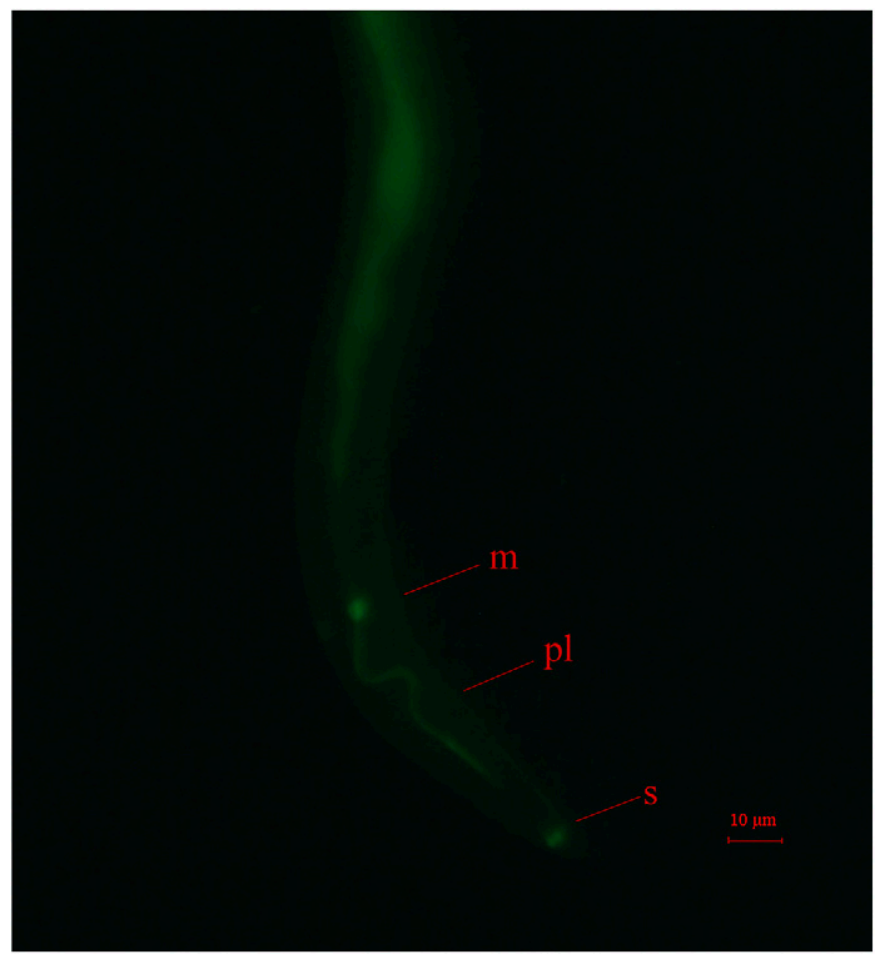

Fig. 2. Fluorescence microscopy showing FITC uptake by Bursaphelenchus xylophilus. Fluorescence was apparent in the pharyngeal lumen and the median bulb. $\mathrm{s}=$ stylet; $\mathrm{pl}=$ pharyngeal lumen; $\mathrm{m}=$ median bulb. indicated that the 36-h treatment period was most effective at silencing Bx-tlp- 1 (Fig. 1A). Accordingly, the subsequent RNAi experiments involved a 36 -h incubation on a rotator at $25^{\circ} \mathrm{C}$ in darkness. The $B x$-tlp- 1 transcript level was $\approx 90 \%$ lower in the dsTLP1-treated PWNs than in the CK-treated or dsGFP-treated PWNs. There was no significant difference in the $B x$-tlp- 1 expression level between the PWNs treated with CK or dsGFP $(P<$ 0.05 ) (Fig. 1B). Additionally, most of the PWNs survived the RNAi treatment, regardless of the buffer solution (Supplementary Fig. S1). Moreover, the qRT-PCR results indicated that the effects of the dsTLP1 treatment were still observable in the F3 offspring (Fig. 1C).

Evaluation of dsRNA uptake with FITC as a marker. The uptake of dsRNA by the PWNs was assessed by fluorescence microscopy, with FITC as a marker. Nematodes treated with dsTLP1 were curled and formed aggregates (Supplementary Fig. S2). Obvious FITC signals were detected in the stylet, pharyngeal lumen, and median bulb of nematodes treated with dsTLP1 (Fig. 2). There was no difference between the PWNs treated with solutions with or without neurostimulants (e.g., octopamine), implying that neurochemicals are not required for the PWNs to take-up FITC. The treatment time was the decisive factor influencing FITC uptake, with fluorescence detected in most nematodes treated for $36 \mathrm{~h}$. These results were consistent with those of a previous study (Cheng et al. 2010).

Influence of silencing $B x$-tlp-1 on $B$. xylophilus reproduction. After culturing $B$. cinerea, the PWN populations at each timepoint $(4,8,12,16$, and 20 days) were counted. The dsTLP1 treatment resulted in fewer nematodes than the control treatment, especially at 20 days (Fig. 3A). The population structure for each timepoint revealed that most of the PWNs were at the larval stage (Fig. 3B). These results implied that the silencing of $B x$-tlp- 1 markedly affects B. xylophilus reproduction.

Symptoms of $P$. massoniana inoculated with $B$. xylophilus. Diverse symptoms were observed on inoculated $P$. massoniana trees, depending on the solution used to treat B. xylophilus. At 27 days after inoculation, the trees inoculated with PWNs treated with the CK or dsGFP solutions had an infection rate of $100 \%$ and a DSI of 100 or 97.5 , whereas the trees inoculated with dsTLP1-treated PWNs had an infection rate of $83.3 \%$ and a DSI of 43.3 (Table 2). At 30 days after inoculations, all of the $P$. massoniana trees were infected, but the trees inoculated with dsTLP1-treated PWNs had the lowest DSI (56.7). Additionally, almost all of the trees
A

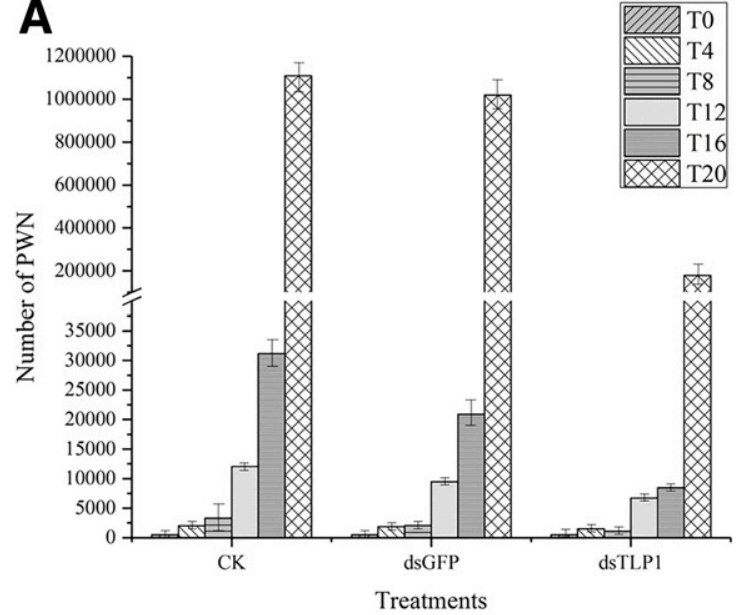

B

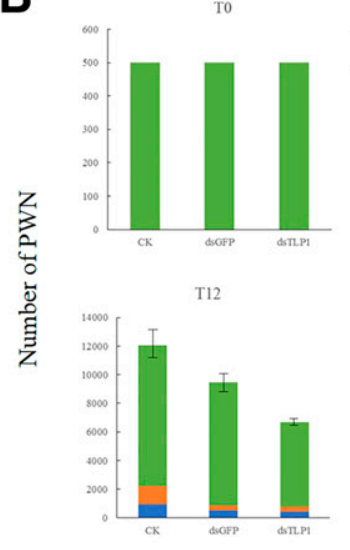

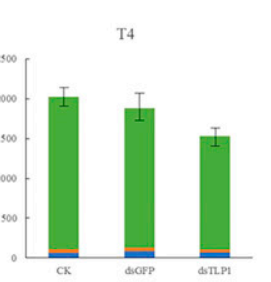

T16

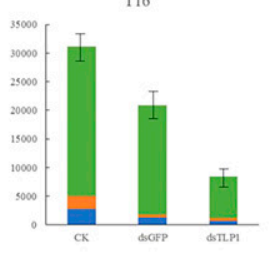

Treatments
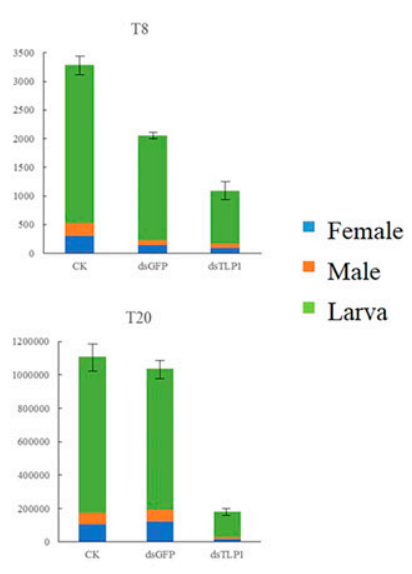

Larva

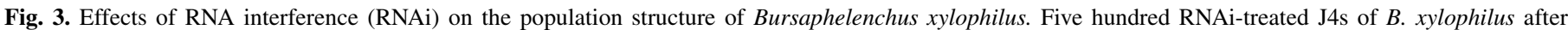

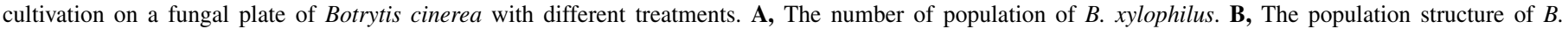

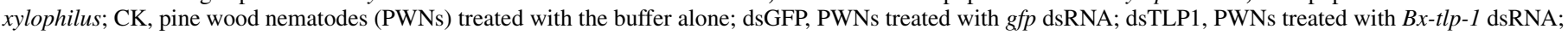

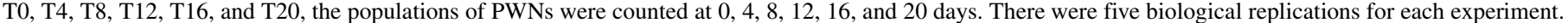
The error line indicates the standard deviation of the mean. 
inoculated with CK-treated or dsGFP-treated PWNs were completely wilted. In contrast, the trees inoculated with sterile water remained healthy (Fig. 4). These results suggested that silencing $B x$-tlp- 1 expression decreases the pathogenicity of PWNs.

Cavitation analysis. At 30 days after inoculation, changes to the longitudinal sections of the phloem and xylem were observable by the eye (Fig. 5). The stem segments of trees treated with sterile water were healthy, buts half of the pith tissue was necrotic and had turned black in trees inoculated with dsTLP1-treated PWNs. Moreover, all of the phloem and pith tissue of the xylem core were completely necrotic and black in trees inoculated with CK-treated or dsGFP-treated PWNs (Fig. 5).

Pine stem samples collected from 2 to $3 \mathrm{~cm}$ below the inoculation sites were analyzed by micro-CT. The two-dimensional crosssections were reconstructed to reveal functional (gray) and air-filled (black) resin and tracheids. The cross-sections of the stem samples from trees treated with sterile water were gray, indicating that resin and tracheids were filled with liquid and functional. However, the cross-sections of samples from trees inoculated with CK-treated or dsGFP-treated PWNs appeared black, implying resin and tracheids were filled with air and that the pith cells had ruptured to form a large cavity. In contrast, the cross-sections of stem samples collected from trees inoculated with dsTLP1-treated PWNs were half gray and half black, reflecting the partial cavitation of the resin and tracheids (Fig. 5). Additionally, the xylem cavitation area of pine trees inoculated with B. xylophilus treated with Bx-tlp-1 dsRNA was $0.46 \mathrm{~mm}^{2}$ at 30 days after inoculation (Fig. 5).

TABLE 2. Symptoms of Pinus massoniana caused by different treatments ${ }^{\mathrm{a}}$

\begin{tabular}{|c|c|c|c|c|c|c|}
\hline \multirow{2}{*}{$\begin{array}{l}\text { Time of } \\
\text { inoculation } \\
\text { (days) }\end{array}$} & \multicolumn{2}{|c|}{ CK } & \multicolumn{2}{|c|}{ dsGFP } & \multicolumn{2}{|c|}{ dsTLP1 } \\
\hline & $\begin{array}{l}\text { Infection } \\
\text { rate }(\%)\end{array}$ & DSI & $\begin{array}{l}\text { Infection } \\
\text { rate }(\%)\end{array}$ & DSI & $\begin{array}{l}\text { Infection } \\
\text { rate }(\%)\end{array}$ & DSI \\
\hline 15 & 50.0 & 33.3 & 46.7 & 32.5 & 6.7 & 1.7 \\
\hline 21 & 66.7 & 61.7 & 66.7 & 62.5 & 56.7 & 25.8 \\
\hline 27 & 100 & 100 & 100 & 97.5 & 83.3 & 43.3 \\
\hline 30 & 100 & 100 & 100 & 99.2 & 100 & 56.7 \\
\hline
\end{tabular}

a Pines inoculated with $\mathrm{CK}=$ pine wood nematodes (PWNs) treated with the buffer alone; dsGFP = PWNs treated with gfp dsRNA; dsTLP1 = PWNs treated with $B x$-tlp- 1 dsRNA. DSI = disease severity index.

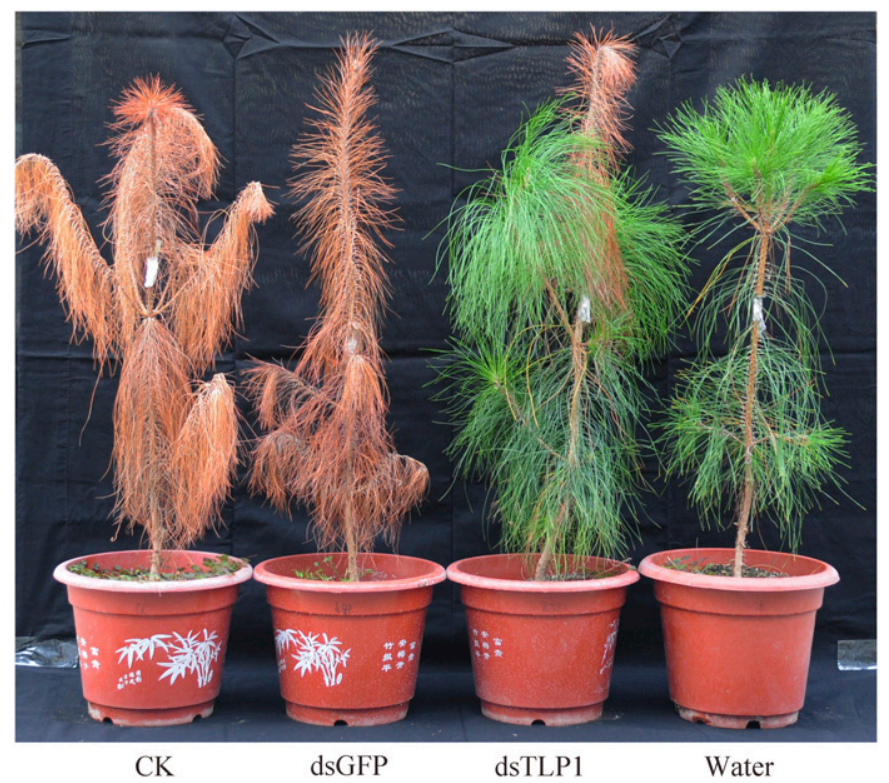

Fig. 4. Symptoms of Pinus massoniana inoculated with different treatments after 30 days of inoculation. Pines inoculated with: CK, pine wood nematodes (PWNs) treated with the buffer alone; dsGFP, PWNs treated with $g f p$ dsRNA; dsTLP1, PWNs treated with $B x$-tlp- 1 dsRNA; and water, sterile water.
Regarding the three-dimensional reconstruction, the $\mathrm{X}-\mathrm{Z}$ and Z-Y sections of the sterile water-treated stem samples revealed intact xylem and phloem tissues with cells arranged in rows and no projective image noise. However, projective image noise was detected in the $\mathrm{X}-\mathrm{Z}$ and $\mathrm{Z}-\mathrm{Y}$ sections of the stem samples from trees inoculated with CK-treated or dsGFP-treated PWNs, and the cells were somewhat disordered. The appearance of the corresponding sections of the stem samples from trees inoculated with dsTLP1-treated PWNs was between that of the sterile water-treated control and the sample from trees inoculated with dsGFP-treated PWNs (Fig. 6). Similar results were obtained for the three-dimensional reconstructions and the two-dimensional cross-sections.

\section{DISCUSSION}

In this study, silencing $B x$-tlp- 1 expression adversely affected B. xylophilus reproduction and pathogenicity. We assessed the effects of soaking PWNs in a dsRNA-containing solution. The dsRNA was taken-up by the PWNs, even in the absence of a neurochemical, which was similar to the results of a previous investigation (Cheng et al. 2010) in which the target gene expression level decreased by $70 \%$ following RNAi induced by soaking samples in buffers supplemented with dsRNA $(1.5 \mathrm{mg} / \mathrm{ml})$. In our study, treatments with dsRNA-containing buffers $(2 \mathrm{mg} / \mathrm{ml})$ silenced the target gene (Bx-tlp- 1$)$ by more than $90 \%$. Additionally, a qRT-PCR assay indicated that the effects of dsTLP1 treatment were maintained in the F3 offspring. RNAi treatment of $C$. elegans via the injection of dsRNA affects nontreated progeny. When genes were targeted by RNAi, the silencing responses were observed for at least three generations (until the F3 offspring). Therefore, these effects may qualify as transgenerational heritable responses (Rechavi and Lev 2017).

Variations in the PWN populations indicated there were fewer dsTLP1-treated nematodes than control-treated nematodes, especially at 20 days. Additionally, there was a measurable decrease in reproduction after the RNAi treatment, suggesting a decrease in nematode fitness. Thaumatin-like proteins exhibit (1, 3)$\beta$-glucanase activity and may bind to $(1,3)-\beta$-glucans in plants. Moreover, TLPs may be capable of binding the cell wall $(1,3)$ $\beta$-glucans of fungi (Osmond et al. 2001). We speculated that the (1, 3 - $\beta$-glucanase activity decreased in dsTLP1-treated PWNs, and that these nematodes could only weakly feed on fungi after RNAi treatment, which may have contributed to the decrease in PWN

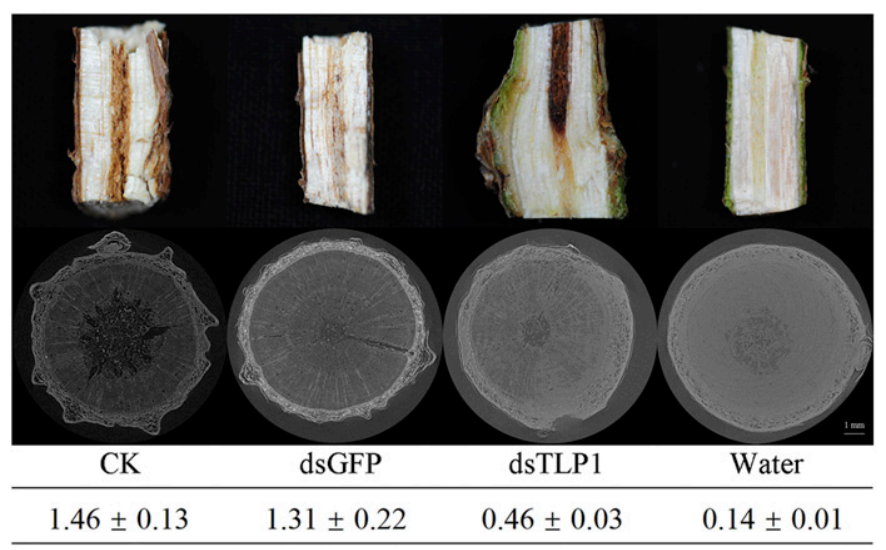

Fig. 5. Internal change of Pinus massoniana after inoculation and direct visualization of xylem cavitation by X-ray microtomography (micro-CT) technology. The scale bar of two-dimensional (2D) cross-sections was $1 \mathrm{~mm}$. The xylem cavitation areas $\left(\mathrm{mm}^{2}\right)$ of $P$. massoniana were measured by Image J. Pines inoculated with: CK, pine wood nematodes (PWNs) treated with buffer alone; dsGFP, PWNs treated with $g f p$ dsRNA; dsTLP1, PWNs treated with $B x$-tlp- 1 dsRNA; and water, sterile water. Data in the table are presented as mean $\pm \mathrm{SD}$. 
reproduction. These results indicated that silencing Bx-tlp- 1 expression adversely affected $\mathrm{PWN}$ reproduction.

Three-year-old P. massoniana trees were inoculated with PWNs treated with CK, dsGFP, or dsTLP1. At 30 days after inoculation, 100\% infection rates were recorded for all inoculated $P$. massoniana trees, but the lowest DSI (56.7) was calculated for the trees inoculated with dsTLP1-treated PWNs. Moreover, almost all of the trees inoculated with CK-treated or dsGFP-treated PWNs were completely wilted. Therefore, B. xylophilus pathogenicity appears to be influenced by $B x-t l p-1$.

A previous study confirmed that the browning of the pith tissue of Pinus thunbergii infected with B. xylophilus for 5 days was observable with the eye (Sheng-Rong and Jian-Ren 2007). Cavitation is a characteristic symptom of PWD that may occur in infected trees in diverse ways (Ikeda and Ohtsu 1992; Utsuzawa et al. 2005). In our study, we measured the cavitation of intact pine stems using microCT. Analyses of the stem cross-sections of trees inoculated with CKtreated or dsGFP-treated PWNs revealed extensive air-filled areas, with ruptured pith tissue cells forming relatively large cavities. However, incomplete cavitation was observed in the stem crosssections of trees inoculated with dsTLP1-treated PWNs. Therefore, the silencing of $B x$-tlp- 1 via dsTLP1 treatment inhibited the ability of B. xylophilus to induce cavitation in pine trees at 30 days after inoculation. The xylem cavitation area of pine trees inoculated with B. xylophilus treated with $B x$-tlp- 1 dsRNA was $0.46 \mathrm{~mm}^{2}$ at 30 days after inoculation. These results implied that $B x$-tlp- 1 is crucial for B. xylophilus pathogenicity, and RNAi may be used to decrease $B x$-tlp-1 expression during infections by PWNs. A previous study confirmed the utility of micro-CT for examining xylem cavitation in
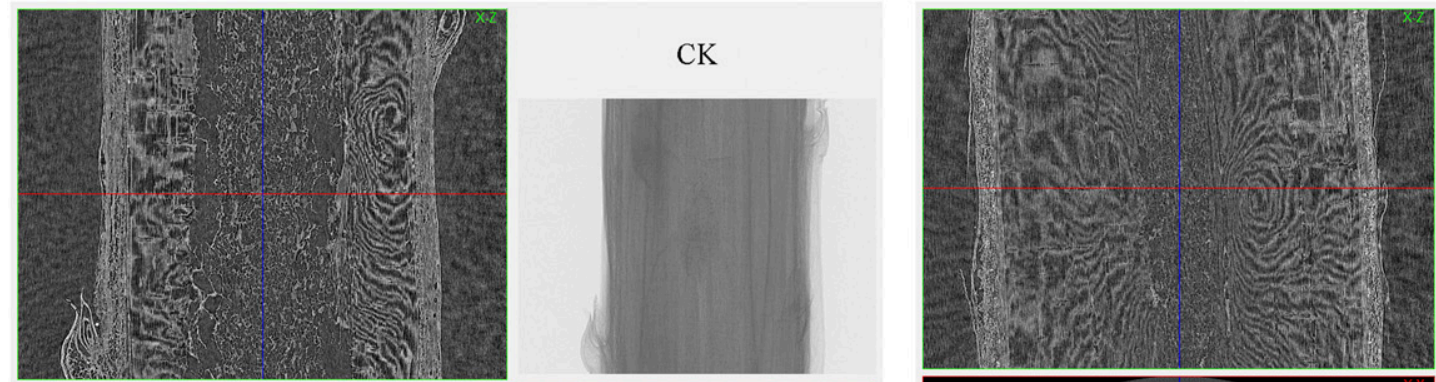

dsGFP
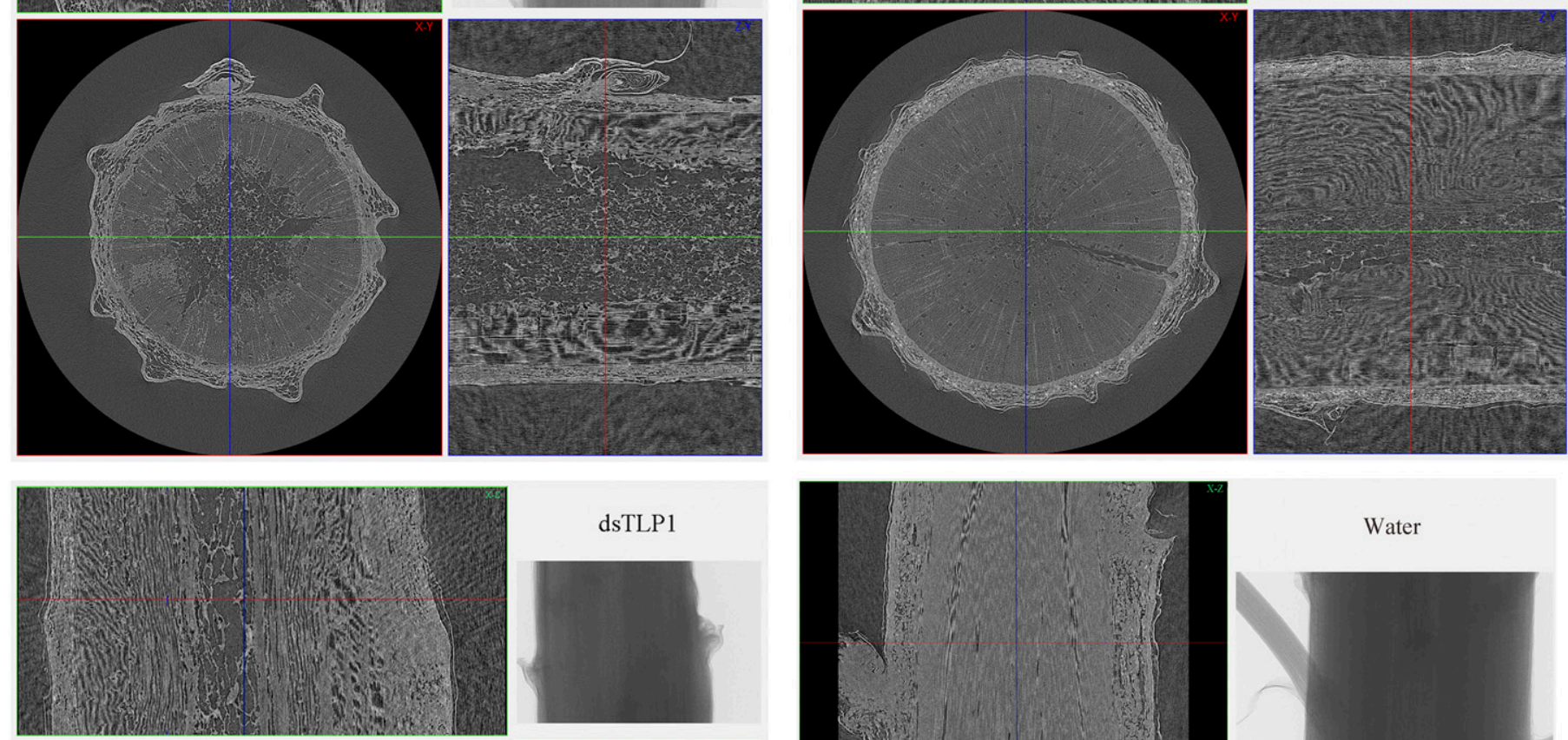

dsTLP1
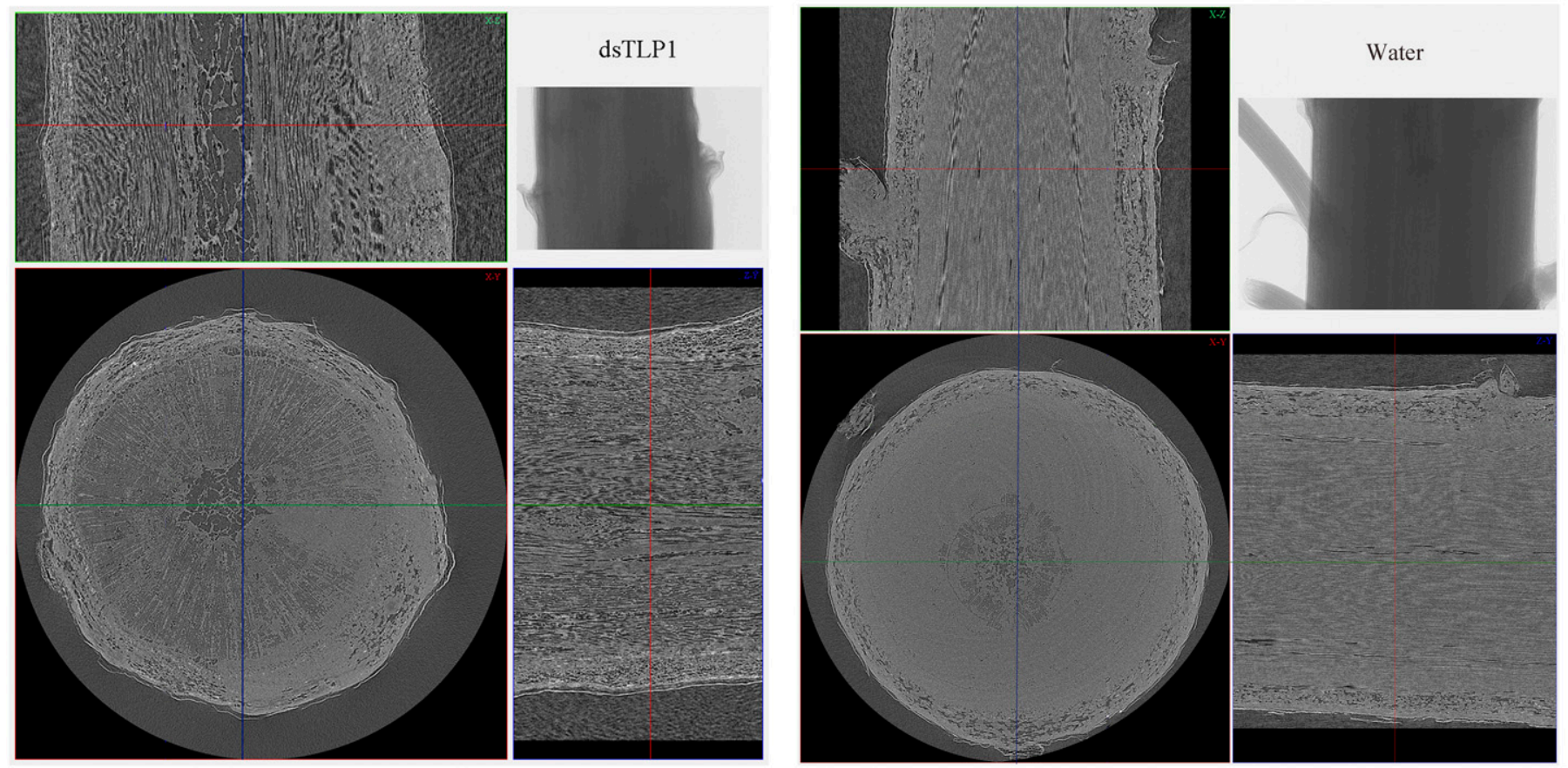

Fig. 6. Three-dimensional (3D) reconstruction (X-Y, X-Z, and Z-Y) of X-ray microtomography (micro-CT). The spatial resolution of the image was $2.5 \times 2.5 \times 2.5 \mu \mathrm{m}$ per voxel. Pines inoculated with: CK, pine wood nematodes (PWNs) treated with the buffer alone; dsGFP, PWNs treated with $g f p$ dsRNA; dsTLP1, PWNs treated with $B x$-tlp-1 dsRNA; and water, sterile water. 
intact samples (Cochard and Delzon 2013). Consequently, micro-CT may be an ideal method for studying cavitation during infections by PWNs. In future studies, we will use micro-CT to further analyze the cavitation of pine trees inoculated with PWNs to clarify the pathogenicity of PWNs.

Previous studies suggested that the protein encoded by $B x-t l p-1$ is similar to the TLPs of $P$. massoniana regarding sequence and structure (Meng et al. 2017; Shinya et al. 2013; Wang et al. 2014). Molecular mimicry is an effective means for plant-parasitic nematodes to evade plant host defenses (Maizels and Yazdanbakhsh 2003). Additionally, Brugia malayi can suppress human macrophages by releasing a secreted homolog of the human cytokine macrophage migration inhibitory factor (Falcone et al. 2001). Moreover, Globodera rostochiensis secretes a CLAVATA3/ESR peptide that affects the host root meristem cells (Mitchum et al. 2012; Wang et al. 2011), which benefits the nematode in plantparasite interactions. Accordingly, the similarity of the protein encoded by $B x$-tlp- 1 to plant proteins suggests it may mimic a component of host plant defense systems. B. xylophilus may trigger defense responses that lead to xylem cavitation. Additionally, a $B$. xylophilus TLP may function as an effector molecule that manipulates plant hosts. Furthermore, the protein may have resulted from the co-evolution of $B$. xylophilus and pine trees or the co-evolution of a close ancestor of B. xylophilus and its hosts.

In conclusion, Bx-tlp- 1 is crucial for B. xylophilus, not only because it influences nematode reproduction but also because it influences the extent of PWD symptoms. The results of this study provide new insight relevant for future characterizations of Bx-tlp- 1 functions related to the interaction between $B$. xylophilus and host pine trees.

\section{ACKNOWLEDGMENTS}

We thank all anonymous reviewers for insightful and constructive comments regarding early versions of this manuscript.

\section{LITERATURE CITED}

Abelleira, A., Picoaga, A., Mansilla, J. P., and Aguin, O. 2011. Detection of Bursaphelenchus xylophilus, causal agent of pine wilt disease on Pinus pinaster in northwestern Spain. Plant Dis. 95:776.

Bakhetia, M., Charlton, W., Atkinson, H. J., and Mcpherson, M. J. 2005. RNA interference of dual oxidase in the plant nematode Meloidogyne incognita. Mol. Plant-Microbe Interact. 18:1099-1106.

Branco, M., Bragança, H., Sousa, E., and Phillips, A. J. 2014. Pests and diseases in Portuguese forestry: Current and new threats. Forest Context and Policies in Portugal. Chapter 5:126-129.

Brandazza, A., Angeli, S., Tegoni, M., Cambillau, C., and Pelosi, P. 2004. Plant stress proteins of the thaumatin-like family discovered in animals. FEBS Lett. 572:3-7.

Cheng, X. Y., Dai, S. M., Luo, X., and Xie, B. Y. 2010. Influence of cellulase gene knockdown by dsRNA interference on the development and reproduction of the pine wood nematode, Bursaphelenchus xylophilus. Nematology 12:225-233.

Cochard, H., and Delzon, S. 2013. Hydraulic failure and repair are not routine in trees. Ann. For. Sci. 70:659-661.

Cochard, H., Delzon, S., and Badel, E. 2015. X-ray microtomography (microCT): A reference technology for high-resolution quantification of xylem embolism in trees. Plant Cell Environ. 38:201-206.

Dubreuil, G. 2007. Transcriptome analysis of root-knot nematode functions induced in the early stages of parasitism. New Phytol. 176: 426-436.

Falcone, F. H., Loke, P., Zang, X., Macdonald, A. S., Maizels, R. M., and Allen, J. E. 2001. A Brugia malayi homolog of macrophage migration inhibitory factor reveals an important link between macrophages and eosinophil recruitment during nematode infection. J. Immunol. 167: 5348-5354.

Fire, A., Xu, S. Q., Montgomery, M. K., Kostas, S. A., Driver, S. E., and Mello, C. C. 1998. Potent and specific genetic interference by double-stranded RNA in Caenorhabditis elegans. Nature 391:806-811.

Gheysen, G., and Vanholme, B. 2007. RNAi from plants to nematodes. Trends Biotechnol. 25:89-92.
Goellner, M., Smant, G., Boer, J. M. D., Baum, T. J., and Davis, E. L. 2000. Isolation of beta-1,4-endoglucanase genes from Globodera tabacum and their expression during parasitism. J. Nematol. 32:154-165.

Grenier, J., Potvin, C., and Asselin, A. 2000. Some fungi express beta-1,3glucanases similar to thaumatin-like proteins. Mycologia 92:841-848.

Hoffmann-Sommergruber, K. 2002. Pathogenesis-related (PR)-proteins identified as allergens. Biochem. Soc. Trans. 30:930-935.

Ikeda, T., and Ohtsu, M. 1992. Detection of xylem cavitation in fieldgrown pine trees using the acoustic emission technique. Ecol. Res. 7: 391-395.

Kitajima, S., and Sato, F. 1999. Plant pathogenesis-related proteins: Molecular mechanisms of gene expression and protein function. J. Biochem. $125: 1-8$.

Luo, J., Li, Z., Ma, C., Zhang, Z., Hull, J. J., Lei, C., Jin, S., and Chen, L. 2017. Knockdown of a metathoracic scent gland desaturase enhances the production of (E)-4-oxo-2-hexenal and suppresses female sexual attractiveness in the plant bug Adelphocoris suturalis. Insect Mol. Biol. 26: 642-653.

Maizels, R. M., and Yazdanbakhsh, M. 2003. Immune regulation by helminth parasites: Cellular and molecular mechanisms. Nat. Rev. Immunol. 3:733-744.

Mamiya, Y. 1988. History of pine wilt disease in Japan. J. Nematol. 20: 219-226.

Meng, F. L., Wang, J., Wang, X., Li, Y. X., and Zhang, X. Y. 2017. Expression analysis of thaumatin-like proteins from Bursaphelenchus xylophilus and Pinus massoniana. Physiol. Mol. Plant Pathol. 100:178-184.

Mitchum, M. G., Wang, X., Wang, J., and Davis, E. L. 2012. Role of nematode peptides and other small molecules in plant parasitism. Annu. Rev. Phytopathol. 50:175-195.

Morimoto, K., and Iwasaki, A. 1972. Role of Monochamus alternatus (Coleoptera: Cerambycidae) as a vector of Bursaphelenchus lignicolus (Nematoda: Aphelenchoididae). J. Jpn. For. Soc. 54:177-183.

Mota, M., Oliveira, S., Vicente, C., and Vieira, P. 2009. Pine wilt disease in Portugal: Recent progress and new approaches to the understanding and control of the pinewood nematode and its insect vector. J. Nematol. 41:361.

Nickle, W. R., Golden, A. M., Mamiya, Y., and Wergin, W. P. 1981. On the taxonomy and morphology of the pine wood nematode, Bursaphelenchus xylophilus (Steiner \&Buhrer 1934) Nickle 1970. J. Nematol. 13: 385-392.

Osmond, R. I. W., Hrmova, M., Fontaine, F., Imberty, A., and Fincher, G. B. 2001. Binding interactions between barley thaumatin-like proteins and $(1,3)$ $\beta$-D-glucans. Eur. J. Biochem. 268:4190-4199.

Rechavi, O., and Lev, I. 2017. Principles of transgenerational small RNA inheritance in Caenorhabditis elegans. Curr. Biol. 27:R720-R730.

Rosso, M. N., Dubrana, M. P., Cimbolini, N., Jaubert, S., and Abad, P. 2005. Application of RNA interference to root-knot nematode genes encoding esophageal gland proteins. Mol. Plant-Microbe Interact. 18: 615-620.

Ryss, A., Vieira, P., Mota, M., and Kulinich, O. 2005. A synopsis of the genus Bursaphelenchus Fuchs, 1937 (Aphelenchida: Parasitaphelenchidae) with keys to species. Nematology 7:393-458.

Shinya, R., Morisaka, H., Kikuchi, T., Takeuchi, Y., Ueda, M., and Futai, K. 2013. Secretome analysis of the pine wood nematode Bursaphelenchus xylophilus reveals the tangled roots of parasitism and its potential for molecular mimicry. PLoS One 8:e67377.

Sakamoto, Y., Watanabe, H., Nagai, M., Nakade, K., Takahashi, M., and Sato, T. 2006. Lentinula edodes tlg1 encodes a thaumatin-like protein that is involved in lentinan degradation and fruiting body senescence. Plant Physiol. 141:793-801.

Shatters, R. G., Boykin, L. M., Lapointe, S. L., Hunter, W. B., and Weathersbee, A. A. 2006. Phylogenetic and structural relationships of the PR5 gene family reveal an ancient multigene family conserved in plants and select animal taxa. J. Mol. Evol. 63:12-29.

Sheng-Rong, S. U., and Jian-Ren, Y. E. 2007. Study on dispersal behavior of pine wood nematode and water potential inside resistant Pinus thunbergii. J. Nanjing For. Univ. 31:125-127.

Sindhu, A. S., Maier, T. R., Mitchum, M. G., Hussey, R. S., Davis, E. L., and Baum, T. J. 2009. Effective and specific in planta RNAi in cyst nematodes: Expression interference of four parasitism genes reduces parasitic success. J. Exp. Bot. 60:315-324.

Sun, Y. 1982. Pine wood nematode found at Dr. Sun Yat's Mausoleum in Nanjing. J. Jiangsu For. Sci. Technol. 4:47.

Togashi, K., and Shigesada, N. 2006. Spread of the pinewood nematode vectored by the Japanese pine sawyer: modeling and analytical approaches. Popul. Ecol. 48:271-283.

Urwin, P. E., Lilley, C. J., and Atkinson, H. J. 2002. Ingestion of doublestranded RNA by preparasitic juvenile cyst nematodes leads to RNA interference. Mol. Plant-Microbe Interact. 15:747-752. 
Utsuzawa, S., Fukuda, K., and Sakaue, D. 2005. Use of magnetic resonance microscopy for the nondestructive observation of xylem cavitation caused by pine wilt disease. Phytopathology 95:737-743.

Vicente, C., Espada, M., Vieira, P., and Mota, M. 2012. Pine wilt disease: A threat to European forestry. Eur. J. Plant Pathol. 133:89-99.

Wang, J., Han, S., Li, Y., Deng, X., and Zhang, X. 2014. Cloning of TLP-1 gene and prediction of TLP-1 protein structure of Bursaphelenchus xylophilus. J. Sichuan Agric. Univ. 32:305-310.

Wang, J., Replogle, A., Hussey, R., Baum, T., Wang, X., Davis, E. L., and Mitchum, M. G. 2011. Identification of potential host plant mimics of
CLAVATA3/ESR (CLE)-like peptides from the plant-parasitic nematode Heterodera schachtii. Mol. Plant Pathol. 12:177-186.

Yoshimura, A., Kawasaki, K., Takasu, F., Togashi, K., Futai, K., and Shigesada, N. 1999. Modeling the spread of pine wilt disease caused by nematodes with pine sawyers as vector. Ecology 80: 1691-1702.

Yu, L. Z., Wu, X. Q., Ye, J. R., Zhang, S. N., and Wang, C. 2012. NOSlike-mediated nitric oxide is involved in Pinus thunbergii response to the invasion of Bursaphelenchus xylophilus. Plant Cell Rep. 31: 1813-1821. 